\title{
TOXSAMPLER: LOCATIVE SOUND ART EXPLORATION OF THE TOXIC RELEASE INVENTORY
}

\author{
Michael V. Blandino \\ Center for Computation and Technology \\ School of Music \\ Louisiana State University \\ mblandi@lsu.edu
}

\begin{abstract}
Regulatory geographic datasets that inform citizen's lives are, in general, responsive to engaged search and visual, attentive browsing, but are not designed for directly informing the lived context. The density of sensors and software interfaces present in mobile devices allows for integration of these resources with contextual applications. ToxSampler is an iOS application that modifies the immediate environmental audio scene with associated data from the Toxic Release Inventory (TRI) of the United States Environmental Protection Agency. The application applies digital signal processing (DSP) to the microphone signal based upon the location of the participant and associated TRI data releases. The system, as a result, affords an informed awareness of the datascape through an immediate augmentation of the sensed setting.
\end{abstract}

\section{INTRODUCTION}

Extremely large data sources describing comprehensive, annual releases of toxic industrial pollution for the United States are difficult to comprehend or to utilize due to their size, dimensionality, ranges of specificity, and the complex methodologies of their collection and manipulation. Accordingly, one must consult separately stored column descriptions or indexes in order to know what data are presented in particular comma-delimited zones of a set. Further, data are significantly abstracted from the setting in which people live, work, and play, by associating values with coordinates of facility identification numbers or in referenced data grids improved by sophisticated, yet rearranged, GIS projections as backend databases for maps. The Toxic Release Inventory (TRI) is such a data source. Helpful visualizations of its data as maps are available directly from governmental institutional sources[1], but even visual information is removed from experience if presented in an aerial, illustrated view that is both uncommon for the average person and difficult to connect to lived experience in a personal, narrative scope. These maps are primarily available in browser-based applets, but the National Institutes of Health (NIH) has recently provided an iOS application to find facilities and release information, bringing the data in closer relation to the user location.

Beyond informational presentations, the increasing ubiquity of mobile devices provides an opportunity for the diffusion of sound

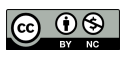

This work is licensed under Creative Commons Attribution Non Commercial 4.0 International License. The full terms of the License are available at http://creativecommons.org/licenses/by-nc/4.0 art through a locative system that may inform participants of their proximity to toxic release activity documented in the historical record of this federal regulatory program. Mobile devices offer high quality components for the realization of such a system: GPS, microphone, audio output, and software interfaces for accessing data feeds from these components. Beyond the halls of a museum or concert hall, an interactive engagement with art informed by these data is possible at locations chosen by or incidental to the participant. ToxSampler is a realization of such a system.

\section{MOTIVATION}

Initially, the intention was to provide a processed mediation between music played by applications on the device and line output, affecting playback. However, limitations preventing access to the music stream of proprietary applications block the routing necessary for this signal processing design. These limitations, though, pointed a way toward processing the available microphone audio stream, an approach which creates an interaction with the actual sounds of the landscape and opens possibilities for augmented audio using the toxic release data.

ToxSampler engages the participant with the TRI release data proximal to their location at a resolution of $2000 \mathrm{~m}^{2}$ by applying audio effects to the microphone input, thereby immersing participants in the data space. There are contours to the release data by location, and salient indicators of their values are not otherwise available while traversing the landscape without referring to visual cartographic resources that display facility location markers and associated release quantities or, at best, shade regions by entire counties to display health risk. Signal processing of the sounds of the present environment based upon values in these data informs the perception of the participant, providing information, but also providing an augmented experience within the senses that can invoke an emotional response. As part of a human/computer system, participants perceive, through their augmented cyborg senses[2], the estimated health risks of their environment that cannot otherwise be perceived.

Use of the personal mobile device empowers the individual to explore spaces where they may incidentally be active. If one were to arrive at a new location for some purpose, querying the risk factors through existing resources on an informative map may give a reference and expectation, but to hear features of the data and experience its contours directly connects one's perception and the choice of one's location to what would not be discernible otherwise. Readily accessible, the mobile device and the application together allow one to incorporate awareness of the underlying en- 
vironmental situation of a place.

\section{BACKGROUND}

Processing an audio signal with control parameters affected by data is a form of sonification or auditory display. The state of this practice lies between poles of tool-making in the service of scientific inquiry and the making of music or sound art informed by data. Much discourse about the mapping of data to sound features emphasizes discernibility, and suggests using high and low pitch levels as preferred options.[3] These recommendations implicitly assume that the primary goal of sonification is primarily for fidelity of display.

Applications of auditory display to data collected in monitoring environmental quality have been realized before. Sounds mapped to air quality data have been synthesized with an orientation toward clarity or even basic representation, using metaphors of sensor instrumentation, such as the Geiger counter or heart monitor[4]. Andrea Polli's New York Sound Map (2006) provided an interactive interface for listening to sounds of the city in an exploration of the sound space. This work includes an engagement with the city as it has manifested and with the features of its soundscape. Her Airlight Taipei [5, 203-250] (2006) project featured air quality sensor readings in a visual and sonic art display, although available only in a fixed location as an exhibition. Similarly, Knowbotic Research's Dialogue with the Knowbotic South [6, 83-86] (1994-1997), which was a seminal work of visual and auditory display of ecological data with impressive visual clarity for the computational capabilities available at the time, could only be explored in the exhibition setting.

A more variable sampling of air quality was realized in the PigeonBlog [7, 192-199] (2006) of Beatriz da Costa, where the sampling was initially controlled by site selection for the release of sensor-equipped pigeons, but with distribution patterns left to the animals from that point. Air quality sensors equipped with GPS components were attached to pigeons who were released to collect data at sites. The website of the project distributed the interface, which included data readings and maps in textual and visual displays.

There is a strong tradition in new media art of making unseen forces or phenomena perceivable through display of gathered data. Greg Niemeyer and Chris Chafe's Ping [6, 136] (2001) was a sonic art work that made packets traversing the network of the world-wide-web audible within a fixed outdoor location at a museum. An event-based work, Usman Haque's Sky Ear [6, 222] (2004), made electromagnetic fields visible via a construction of helium balloons configured in connected rings with light emitting electronics to form a large mass with discrete sensors and flashing displays. The Sky Ear detected and gave feedback of electromagnetic activity in the vicinity of the site. Participants could interactively call parts of the apparatus to enable effects. Christina Kubisch's Cloud 2017 [8] features unseen magnetic fields as composed presentations recorded from actual locations. Participants hear unseen yet physically present patterns through a direct sensor interface within the custom headphone apparatus that detects her sculpted playback interface. A more locative and situationally exploratory project, Kubisch's Electrical Walks of San Francisco (2016) brings unseen electromagnetic fields that are active in the cityscape to participants' ears. ToxSampler brings unseen toxic release data together with the soundscape, unifying several threads present in these historical examples, but utilizing the personal, mo-

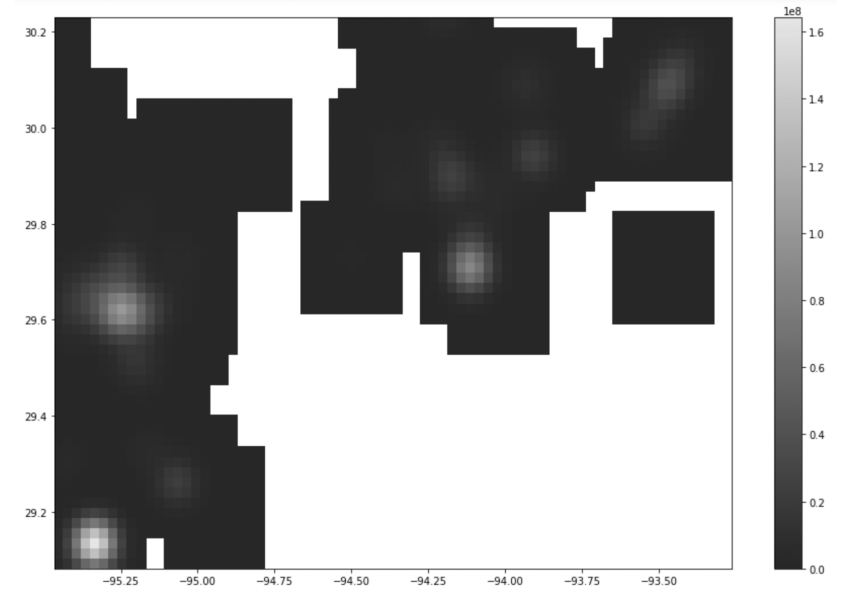

Figure 1: A subsection of the prepared ToxSampler release grid that controls sound parameters

bile device.

\section{TECHNICAL REALIZATION}

The software of the system is programmed as an iOS application with data derived from, at the time of its development, the most recent comprehensive TRI data set from 2015. The participant's location is accessed by the software through the Location Services function persistent within the iOS, which draws upon GPS, cellular, and wireless internet location data. The application will still work when outside of cellular or wireless networks if a GPS chip is present on the device.

ToxSampler is built with the Swift programming language. The release data grid is stored within the application and is imported on build from a gridded summation of release data convolved with a Gaussian kernel to extend meaningful values from release coordinate points. Audio processing makes use of AVAudioEngine effects with control parameters guided by the grid data according to coordinate locations from the location services utility.

Out of respect for privacy, and for conformance to development guidelines enforced by the operating system manufacturer, the participant must grant permission to utilize the microphone as well as to access the location. In the ToxSampler system, these data are strictly used only for the audio signal processing and are not stored persistently beyond the application session, nor are they remotely accessible or transmitted in any way.

\subsection{Augment Reality}

ToxSampler does not provide instrumentation or visual feedback in units on a dial. While the intention is to convey the measured risk of pollution's effects in the locale of the listener, it is through an experiential and representative deformation of the directly sampled audio presence of the locale itself that this risk is presented. One can proceed to explore the joined physical space informed by the processed audio and data space (grounded in actual release data). Periodic location checks update the TRI reference value and change the attendant signal processing values accordingly. Thus, the virtual locale presented by ToxSampler augments reality with the mapped sound design parameters. 
Due to its derivation from release data, the application is informative and augments with truthful observations as reported through the policy regime. In that it informs through manipulation of sensation of the world, the augmentation provided by the application is experiential, changing the sensory system in which the participant perceives the acoustic energy of the immediate sound field. In a perceptual sense, sensation is purposefully degraded by the application to connect the toxic release to the sensory apparatus. Disorientation is a result - one intended to inform, not only by discernible quantified values, but by the qualitative effect on experiencing the world through the mediated deformation.

\subsection{Sound Design and Parameter Mapping}

As opposed to synthesized or direct realization of sampled data into audio signals as is often found in instrumentation-oriented auditory displays, ToxSampler uses the information values as control signals to affect the audio signal from the microphone. The signal processing effects and the data are discernibly related in changes of direction and magnitude.

The echo is a phenomenon of recognizance and of returning sound of perceived events or, in the case of sounds originating from an agent, actions. The sounds return, but encoded within these sounds is information about the event or action and about the resonating structure. The amount of time spanning a natural reverberation or echo is information itself, informing a witness of the dimensional features found in a space. Echo added through ana$\log$ or digital signal processing delay embeds information about a virtual space. ToxSampler adds a digitally realized delay to the microphone signal in units of time (maximum 2 seconds) to partially encode the level of toxic releases in a location. For however long the delay time, the higher the documented release amount is for the given data region. Because the range of release data has large outliers and because significant values are intended to be salient in ToxSampler, a logarithmic scaling is used to set the control parameters.

The gain of the delayed signal (on a scale of 0-100) is set by a mapping, (1),

$$
4 \log _{2}(r+1)
$$

where $r$ is the release sum for the associated location grid.

The delay time in seconds is mapped according to (2).

$$
0.08 \log _{2}(r+1)
$$

The impulse response ${ }^{1}$ of the ToxSampler system to a noise impulse in a recording studio setting is shown in figure 2 . With a local toxic release value yielding a delay of 1.478 seconds, the delay and feedback gain values affect the signal accordingly. Impulse decay from $-3 \mathrm{~dB}$ to $-60 \mathrm{~dB}$ occurs over a span of 29.49 seconds.

Another effect, distortion, causes noise-like influences on the quality of audio signals, introducing textures that deform the sound significantly. Distortion is added to the ToxSampler signal at a scaled level that is increased as the TRI data increases. One cannot compare or recall levels of distortion as clearly as one can a delay time value. Distortion, however, is more suggestive of a degradation of the sound environment which mirrors the degradation of the life-sustaining environment by toxic releases.

${ }^{1}$ The displayed impulse response recording of figure 2 may be accessed at http://blandino.art/ToxSamplerImpulse.wav, along with other audio examples.

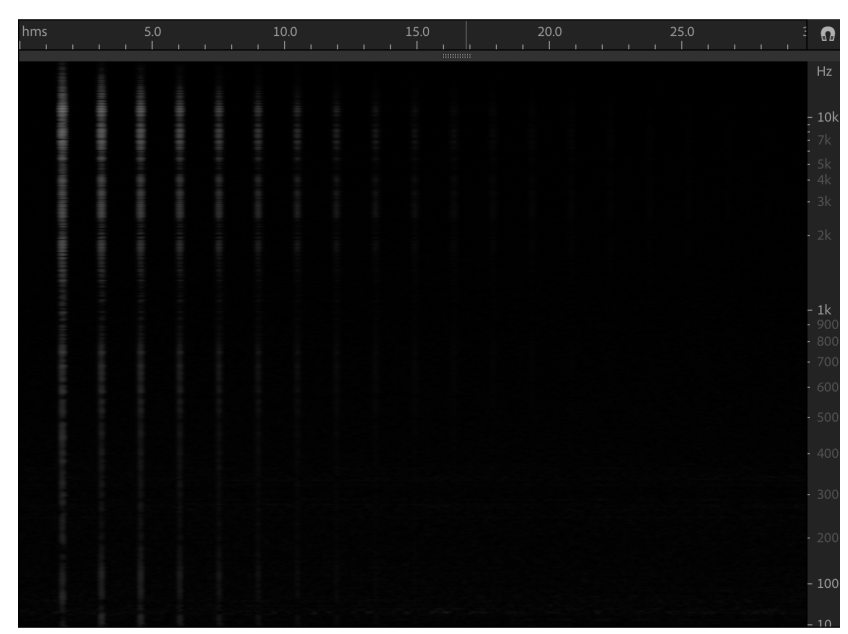

Figure 2: Impulse response of ToxSampler at a location with resulting delay time of $1.478 \mathrm{~s}$

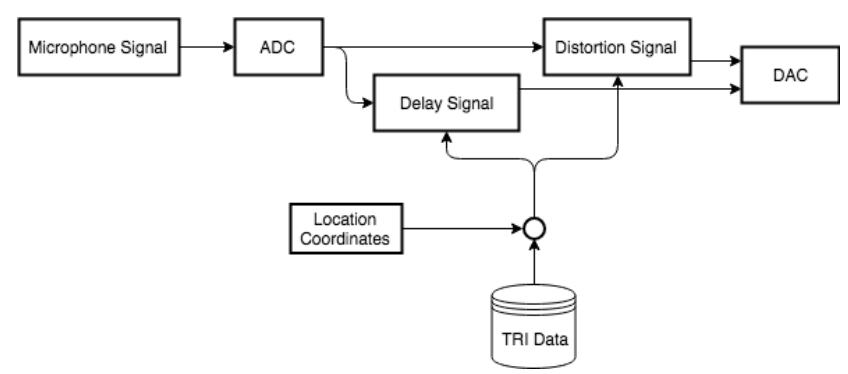

Figure 3: The audio signal flow of the ToxSampler application.

The distortion level (0-100) applied to the incoming signal is mapped according to (3).

$$
0.5 \log _{2}(r+1)
$$

\subsection{User Interface}

Informing participants through sound as opposed to visual cartographic and quantitative display modes, the ToxSampler application does not provide a graphic control surface beyond enabling the app and deactivating it. A demonstration mode is available for presentation and testing settings in order to compare the sound results from an area with high levels of releases vs. another with none or to engage participants who only are allowed a limited interaction with the application by selecting their home location or a favorite (or known release) site.

\section{DISCUSSION}

\subsection{Blending the Real and the Virtual}

Modification of the immediate casts the perception of the user into a virtual, mediated context. In the case of ToxSampler, the context is a virtual one informed by the measurement of personal risk factors to the best level of knowledge available. While virtual, recognizability of the real is preserved, in that sounds may still be 
discerned within the level of distortion set and within the delayed returns. The making unreal of the real and making real of the obscure are operations that support resulting reflection, but they simultaneously dull the impact of the health risk and effects of the toxic discharges on the community of the user or that of the participant's temporary locale as synthetic constructions. On the other hand, the release data are declarations of physical, located releases that were real and are likely to continue in the location alongside the continued, associated industrial activity. The manifestation of the representations of these releases in our perception, however virtual, is sourced in the real.

\subsection{Ethics and Manipulation}

The scale and quality of sonification signal processing must be informed by considerations of design to ensure salience of the data but also by considerations of ethics - out of concern for participant perception, for representing facility actors, and for representation of health risks. It is possible to process an audio signal to such a degree that no sounds are audibly recognizable or at a volume that is directly painful. To do so in this case would sever the link between present environmental experience that connects events and actions in the environment to what is heard through the system. Such an extreme influence of the data space would suggest commensurate extremes of degradation of the environment that would imply that an area is altogether uninhabitable or even instantly, fatally toxic. Thus, recognizability of environmental sounds captured by the microphone is intentionally preserved in the system design.

At the other end of the data domain, where there is zero risk of exposure to toxic releases, the audio signal is passed through with no influence, not even to compensate for the attendant distortions or artifacts inherent to listening to the environment through the small microphone of a mobile device. In the contour of salience that runs between the maximum and minimum, one could err in either underrepresenting or overrepresenting risk in the rate of increase in level of influence caused by the data at various points of the mapping curve.

\subsection{Scenario}

Walking a landscape, be it urban, suburban, rural, or industrial, with headphones and either the built-in microphone or an accessory microphone, the user of ToxSampler will, as a basis, hear the sounds before them. The attendant attributes of listening through a small microphone will be present: everyday sounds are amplified such that the resonance of a space is prominently noticeable and small sounds near the microphone, especially those associated with the user's movement, are noticeable in the soundscape. Dependent on the proximity to documented facility locations with releases in the TRI, the sounds will be colored by the signal processing representative to the data space.

The intention of the listener to sample the data space, and to choose how to move within it, gives agency to introduce a potential for manipulation of the signal and explore the landscape with this manipulation added and, thereby, experiencing it through the mediating "lens" of the dataspace. Unattached to a gallery or fixed terminal or kiosk, the work is mobile, and control of its realization is distributed.

\subsection{Discovery}

ToxSampler does not provide a reference back to what industrial entities are releasing toxic chemicals or any municipal zoning or regulatory influences that allow or encourage their existence. Situationist exploration of the landscape[9] alongside the annotated sound experience is, thereby, an exploration of recorded release events in actual space and of the artifacts and expressions of desire by investors, consumers, and political actors who bring them about. One need not travel excessively far to perceive a gradient increase or decrease in release values due to the resolution of data, if near a release site. Multiple ToxSampler listening experiences in locations that are far apart will provide contrasting experiences in remembered relationship across time.

\subsection{Tactical Action}

There are limitations to the implementation of ToxSampler as a vector for tactical action. It is prepared more readily as a means of informing individual experience with environmental risk factors. Wearing headphones is an isolating gesture not given to community interaction and sharing, but a group could certainly go on a ToxSampling excursion together. To use ToxSampler, one must be in a setting where care can be taken to hear well enough via headphones or amplification, and either take a static ToxSample or move across the static data space. One may record the output of the application quite readily with an auxiliary cable and recording device and share the results or incorporate into derivative art. Listening in a car to traverse more rapidly across the datascape allows one to hear contours in the parameter shifts and introduces the possibility of feedback from loudspeakers. Intervention at a public location could certainly be realized with preloaded devices.

\subsection{Participant Feedback}

Participants who have used ToxSampler report an increase of knowledge about the releases in their locale as a result of engaging with the system. In one instance, an area that a participant assumed would have high release data was discovered to have a null release value. Participants who live and work in an area surrounded by multiple sites with relatively high release levels, the near-continuous alteration of the audio gathered from the environment elicited concern about health risks and about complacency regarding the continuous presence of environmental health risks. Transacting mundane activity such as pedestrian travel while listening to the ToxSampler signal in such an area was reported to be disorienting but informative in an enveloping way.

\subsection{Complicity}

Participating in a human/computer system that includes a highlysophisticated, mass-produced electronic device is an inherent engagement and complicity in toxic releases resulting from the creation of the device and emissions from its energy sources. It is important to note that participants can generally be considered complicit as consumers and, to the extent that their political power may be exercised, willing as citizens to accept the distribution of risks involved with the creation of products that range from the arguably necessary to the disposable. Although ToxSampler does not include global data for toxic releases and, therefore, releases associated with the manufacture of iOS devices outside of the United 
States, the device and its environmental costs as part of the human/computer system must be acknowledged as part of the meaning of the system.

\section{FUTURE WORK}

The ToxSampler makes use of direct release information and does not incorporate water flow, toxicity risk factors, prevailing wind directions, and other important contributing influences on health factors for a given location. Informing the ToxSampler with the Risk-Screening Environmental Indicators (RSEI) data grid is the next phase of development for this project as this data is more directly related to health factors and includes much analytical expertise dedicated to the public interest.

\section{REFERENCES}

[1] TRI and Superfund Environmental Maps - TOXMAP. National Institutes of Health. [Online]. Available: https: //toxmap.nlm.nih.gov/toxmap/

[2] N. Wiener, "Men, machines, and the world about," Medicine and Science, pp. 13-29., 1954.

[3] A. De Campo, "A data sonification design space map," in Proceedings of the 11th International Conference on Auditory Display (ICAD-2007), 06 2007, pp. 342-347.

[4] M. S. Pierre and M. Droumeva, "Sonifying for public engagement: A context-based model for sonifying air pollution data," in Proceedings of the 22nd International Conference on Auditory Display (ICAD-2016), 07 2016, pp. 60-64.

[5] A. Polli, Toxic Airs: Body, Place, Planet in Historical Perspective., A. Johnson and J. R. Fleming, Eds. Pittsburgh, Pa. : University of Pittsburgh Press, 2014.

[6] C. Paul, Digital Art. Thames and Hudson, 2015.

[7] X. Burrough, Net Works : Case Studies in Web Art and Design. New York; London : Routledge, 2012.

[8] R. Frieling, Christina Kubisch. SFMOMA. [Online]. Available: https://www.sfmoma.org/publication/soundtracks/ christina-kubisch/\#cloud

[9] M. d. Certeau, The Practice of Everyday Life. Berkeley : University of California Press, 1984. 